\title{
La didactique du FLS, entre approches communicatives et français de scolarisation
}

\section{Bruno Maurer}

\section{(2) OpenEdition \\ 1 Journals}

\section{Édition électronique}

URL : http://journals.openedition.org/trema/2165

DOI : 10.4000/trema.2165

ISSN : 2107-0997

\section{Éditeur}

Faculté d'Éducation de l'université de Montpellier

\section{Édition imprimée}

Date de publication : 1 juin 1995

Pagination : 13-25

ISSN : 1167-315X

\section{Référence électronique}

Bruno Maurer, "La didactique du FLS, entre approches communicatives et français de scolarisation», Tréma [En ligne], 7 | 1995, mis en ligne le 23 septembre 2013, consulté le 19 avril 2019. URL : http:// journals.openedition.org/trema/2165; DOI : 10.4000/trema.2165

Ce document a été généré automatiquement le 19 avril 2019

Trema 


\title{
La didactique du FLS, entre approches communicatives et français de scolarisation
}

\author{
Bruno Maurer
}

1 L'existence d'un statut particulier réservé au français dans certaines régions du monde et la nécessité de conceptualiser cette place ne font plus aujourd'hui aucun doute. Si la réalité d'un français langue seconde est sociolinguistiquement acquise, même si l'on peut par ailleurs émettre des réserves sur le rôle que joue cette conceptualisation dans la reproduction des situations diglossiques, il semble qu'en matière de didactique aucune avancée notable n'ait été accomplie. Dans un numéro des Études de linguistique Appliquée. M. M. Ngalasso (1992: 35) constatait qu'une didactique spécifique du Français Langue Seconde n'existait pas et, en guise de pistes de recherche, offrait aux lecteurs une série d'interrogations.

2 Force est de constater, avec J.-P. Cuq (1992 : 5), que le concept de français langue seconde a du mal à «entrer dans la panoplie intellectuelle» des chercheurs en didactique. Les didacticiens du français sont actuellement partagés entre deux attitudes dont, à notre avis, aucune ne prend suffisamment en compte la spécificité du français dit langue seconde.

3 L'attitude la plus commune, au moins jusqu'à une date très récente, consistait à ne pas considérer la spécificité didactique du français langue seconde, à lui reconnaître au plus une existence sociolinguistique dans les pays africains par exemple où le français n'est tout à fait ni langue maternelle ni langue étrangère. M. M. Ngalasso (1992 : 30) range au nombre de ces chercheurs des personnalités de premier plan comme R. Galisson et D. Coste (1976) ou E. Roulet $(1975,1980,1986)$. Soit la distinction n'est pas faite entre français langue seconde et français langue étrangère (E. Roulet), soit elle est considérée comme pédagogiquement non justifiée (R. Galisson et D. Coste). Pourtant, les conditions d'apprentissage du FLS, auprès d'un public le plus souvent très jeune, maîtrisant mal sa langue maternelle, très souvent contraint de suivre en français l'enseignement de toutes les autres disciplines, sont très différentes de celles d'une langue étrangère commencée 
souvent à un âge plus tardif, comme matière et non comme média d'enseignement. En cela, il nous semble que l'on ne peut plus faire l'économie d'une réflexion spécifique au FLS, dont la valeur instrumentale doit être reconnue.

4 Cette prise de conscience, somme toute très récente donc, doit être mise au crédit de didacticiens étant ou ayant été au contact du terrain africain, où la question de l'enseignement de la langue seconde se pose avec le plus d'acuité. G. Vigner $(1987,1992)$ et J.-P. Cuq $(1991,1992)$ contribuent ainsi par leurs travaux à changer les mentalités, à faire émerger un champ didactique du FLS.

5 Toutefois, il nous semble que leurs propositions, convergeant autour de l'idée d'une didactique du français comme "langue de scolarisation", sont, en dépit de leurs nombreux mérites, trop réductrices. Pour faire court, disons qu'une trop grande focalisation sur l'aspect français - médium d'enseignement est difficilement réalisable dès les débuts de l'apprentissage en raison de l'âge des apprenants et de la nature des savoirs à enseigner et que l'on peut difficilement se passer totalement, pour un public débutant de cette nature, des approches dites communicatives. C'est donc, dans un premier temps, à une lecture critique de ces travaux visant à une rénovation de la didactique du français que nous voudrions nous livrer, dans le but de leur adjoindre d'autres hypothèses de travail, à notre sens complémentaires. Nous essaierons de déterminer ensuite quel profit les enseignants peuvent tirer de cette réflexion sur le FLS dans leur pratique quotidienne en France, face à un public de migrants pour lesquels le français n'est pas non plus tout à fait la langue maternelle.

\section{Les approches communicatives sont-elles pertinentes?}

6 La démarche des promoteurs d'une didactique du français langue seconde vise, à juste titre, à «maximiser» (M. M. Ngalasso, 1992: 28) le concept de langue seconde. Les principaux défenseurs de ce courant sont G. Vigner (1987 et 1992) et J.-P. Cuq (1991 et 1992). Pour reprendre brièvement l'exposé de leurs positions, on peut dire que ces auteurs rejettent l'utilisation des méthodes d'inspiration communicative pour privilégier une autre dimension de la langue, plus informative que communicative : ils proposent de fonder la didactique du FLS autour du rôle essentiel de cette langue, celui de scolarisation.

7 L'argumentaire relatif à l'utilisation des méthodes communicatives dans les pays où le français est langue seconde, et plus particulièrement en Afrique francophone, ne manque pas de pertinence. G. Vigner (1987: 44) dénonçait l'emploi en Afrique « de méthodes trop actives, qui ignorent les rituels africains de prise de parole, la place de l'enfant dans la société ».

8 À cette première critique, que l'on pourrait qualifier d'ethnographique, s'en ajoute une seconde, plus strictement sociolinguistique : le français ne servant pas à répondre aux situations courantes de communication, le choix de méthodes reposant sur l'utilisation du français dans pareilles situations est une erreur. G. Vigner écrit ainsi (1992: 41) que les «principes communicatifs en FLS ne sont pas valables» pour la simple raison que les apprenants n'ont pas besoin du français pour communiquer, disposant pour ce faire de leur langue maternelle : 
9 «Communiquer clans les situations de la vie courante, apprendre à exercer dans ce cadre un certain nombre de fonctions de communication, c'est faire l'hypothèse que dans l'univers de vie de l'élève, celui-ci ne dispose pas d'un autre outil linguistique. Or en situation de langue seconde l'élève dispose déjà d'une autre langue, sa langue maternelle (...). »

10 J.-P. Cuq dresse le même constat (1991: 191) :

« Il est donc peu probable que l'utilisation de méthodes d'inspiration communicative présente un intérêt majeur dans de telles situations et il semble qu'elles n'aient pas rendu les services qu'on en attendait là où on a tenté de les utiliser. »

11 Sur un plan différent, G. Vigner va jusqu'à affirmer (1992: 43) que la prédominance des approches communicatives, ne permettant de prendre en compte que la seule « fonction de communication (interaction entre deux individus) ", occulte une autre dimension, cognitive et non plus sociale, de la langue seconde. Les auteurs conseillent donc d'insister sur la dimension cognitive du langage et plus sur sa dimension sociale, essentielle dans les méthodes communicatives. Travaillant dans cette direction, G. Barbé (1988: 40) montre que la langue seconde relaie la langue maternelle «et prend en charge l'instrumentalité qui lui revient dans le développement cognitif. L'enfant l'apprend pour apprendre d'autres choses. » Elle note aussi l'importance de la langue seconde dans les processus de construction de l'identité du sujet.

\section{L'hypothèse de l'enseignement d'un français de scolarisation}

12 Cet argument nous amène au cœur de la conception didactique des représentants de ce courant. À quoi sert le français quand les langues maternelles, premières, assurent l'essentiel de la communication sociale ? G. Vigner et J.-P. Cuq répondent, chacun de leur côté, que son utilité est avant tout scolaire. G. Vigner va même jusqu'à faire de ce trait le caractère définitoire minimal du FLS (1992: 40) :

«Langue apprise pour enseigner d'autres matières qu'elle-même et qui peut, dans certains pays, être présente dans l'environnement économique et social des élèves. »

13 Cela le conduit à penser qu'il est plus pertinent de fonder une pédagogie du FLS sur une « sociolinguistique des échanges et usages scolaires de la langue » que sur une " analyse centrée sur les seuls usages sociaux (hors de l'école donc)» (1992: 53). Toujours pour G. Vigner, il existe une « communication spécifique à la communauté scolaire» (1992: 43) qui doit être prise en compte lors de la réflexion didactique, cet auteur allant même jusqu'à parler de « communauté linguistique particulière » formée des écoliers, lycéens et étudiants ayant en commun l'usage du français à l'école. La didactique du FLS doit exercer ces «zones de compétence qui sont liées à l'exercice du travail scolaire » et permettent de communiquer avec succès dans cet univers; elle doit aborder ainsi les «marques organisatrices du discours pédagogique» (1992: 43). On voit que pour G. Vigner, le FLS étant avant tout une langue de scolarisation ${ }^{1}$, de ce constat doivent partir toutes les recherches en matière de didactique.

14 Mais si toutes ces prémices, plus psycho ou sociolinguistiques que réellement didactiques, semblent fondées, leurs applications pratiques en matière d'enseignement du français apparaissent, selon nous, plus discutables. 

«suggestions didactiques »: elle propose de «partir de situations problématiques » au lieu de chercher à se raccrocher à la vie quotidienne des apprenants, dans laquelle la langue maternelle a, presque seule, droit de cité. Mieux vaudrait se référer à des thématiques en relation avec l'univers scolaire, comme les autres matières enseignées. À titre d'exemple, elle avance que l'on peut faire réfléchir les élèves à leur école dans le but d'en faire la présentation à un éventuel visiteur officiel. Cela permet de déterminer une série d'opérations à réaliser, notamment des «actions langagières " comme «se situer dans le temps, dans l'espace, compter, décrire, comparer.» On voit bien comment ces propositions didactiques peuvent découler de considérations sociolinguistiques: le français n'étant généralement parlé qu'à l'école, c'est au sein de cet univers thématique que doit s'effectuer l'apprentissage. On remarquera au passage que, malgré les critiques formulées par ailleurs à l'encontre des méthodes communicatives, le concept de communication authentique, souvent dénoncé comme mythifié, n'a pas été tout à fait évacué.

16 J.-P. Cuq considère de son côté que, dans cet apprentissage scolaire de la langue seconde, « priorité » doit être « donnée aux compétences de décodage » $(1991: 197)$ :

«Or, ce sont justement les compétences de décodage qui priment à notre sens pour une langue seconde qui reste avant tout un moyen informatif et de formation avant d'être un vecteur de communication. Ainsi, à l'oral, il sera peut-être préférable de privilégier, dans un premier temps au moins, la compréhension. »

Comme nous l'avons montré, cette priorité est fondée sur le fait que les besoins communicatifs essentiels de l'enfant sont assurés pour l'essentiel par sa langue maternelle et que la nécessité qui se fait jour relativement au français, c'est avant tout de « comprendre le sens des enseignements qui lui sont dispensés en cette langue » (1991: 198). Bien entendu, J.-P. Cuq ne propose pas de renoncer à développer les capacités d'expression mais d'en décaler l'apprentissage :

Ce n'est qu'au fur et à mesure que se développeront les capacités d'expression que l'enseignant multipliera les situations de communication, en commençant par celles dont les probabilités de réutilisation sont les plus immédiates » (1991:198).

G. Vigner n'est pour sa part guère explicite quant aux moyens d'enseigner cette langue en en privilégiant la fonction de scolarisation; l'idée générale est de s'appuyer sur les situations d'apprentissage elles-mêmes, en tant que "situations productrices de discours donnant lieu à des fonctionnements langagiers spécifiques» (1992: 40-41). La partie intitulée "Aspects de l'apprentissage » ne permet guère de se faire une idée plus précise quant à la manière dont doit se faire l'apprentissage du français. En revanche, une autre idée-force se fait jour: G. Vigner se montre partisan d'une "abondance linguistique » dans laquelle doit baigner l'apprenant lors de l'apprentissage - il utilise l'expression de «bain linguistique » - et rejette l'idée de progressions fondées sur un «calibrage » de la matière enseignée (1992 : 47). 


\section{Le français de scolarisation est-il un concept rentable en termes didactiques?}

20 Arrivé à ce stade de la réflexion, nous voudrions exprimer nos convergences de vue avec une partie des thèses énoncées ci-dessus, même si nous n'en suivons pas les auteurs dans toutes leurs conclusions.

21 Ainsi partageons-nous les présupposés psychocognitifs exposés ci-dessus sur le rôle du FLS dans le développement de l'enfant, les réflexions d'ordre à la fois sociolinguistique et didactique de J.-P. Cuq sur la nature du français à enseigner (1991: 192-195), ou la nécessité de pratiquer une réelle interdisciplinarité dont la langue française serait le centre (Vigner, 1992 : 47-48 ; Cuq, 1991 : 205-206). La priorité donnée à l'écrit (Cuq, 1991 : 204) est également un principe souhaitable dans des pays où le français sert avant tout à la communication écrite et où la maîtrise de ce code conditionne pour l'essentiel la réussite scolaire. Il en va de même pour le principe de l'abondance linguistique, dont nous ferons tout de même remarquer au passage qu'il n'est pas l'apanage d'un français de scolarisation et ne saurait en tous les cas être incompatible avec les approches communicatives. Toutes ces considérations méritent effectivement d'être intégrées à une didactique du FLS.

Pourtant, plusieurs divergences nous empêchent de suivre plus avant les tenants du français de scolarisation défini plus haut comme centre de la didactique du FLS.

1. En premier lieu, on peut dire que si l'idée d'enseigner une sorte de français de spécialité, le français de scolarisation, est bien conforme à la réalité sociolinguistique la plus courante en Afrique, elle ne tient pas assez compte en revanche des motivations des apprenants. Pour séduisante que soit cette hypothèse, elle n'en est pas moins peu réaliste : on n'apprend pas une langue à l'école pour parler de l'école. À trop vouloir tenir compte des besoins langagiers réels, on risque d'en oublier les centres d'intérêt des enfants. Or ceux-ci entrent certainement beaucoup plus en ligne de compte dans la motivation des apprenants que des besoins que, de toute manière, ils ne ressentent qu'exceptionnellement. S'il est indéniable que les besoins de communication, dans les situations où le français est langue seconde, se limitent souvent à la communication intrascolaire (Cuq, 1991 :191), rien ne serait moins motivant pour les apprenants que l'impression de vase clos qui résulterait d'un apprentissage centré sur cet univers. D'une manière générale, les propositions didactiques se réclamant du concept de français de scolarisation, ou de français d'information, sousestiment la nécessité d'impliquer l'apprenant dans son apprentissage, de lui donner rapidement goût à la langue en lui offrant l'occasion de s'exprimer sur des sujets et dans des situations plus motivantes que celles référant à l'univers scolaire. Il nous semble ainsi que, paradoxalement, les tenants du français de scolarisation s'enferment dans un travers des méthodes communicatives que pourtant ils critiquent: celui de l'authenticité. Le français n'a d'utilité sociale qu'en situation scolaire? Centrons son apprentissage, disent-ils, sur cette situation afin de cerner au plus près l'authenticité des échanges et de ne pas tomber dans le piège de la «fictivité ». Mais ce faisant, on court le risque d'accentuer la coupure existant souvent entre l'univers scolaire et le milieu de l'enfant et, partant, de rendre vain son apprentissage. On confond ensuite les données de fait - la limitation actuelle du français à quelques domaines restreints dont l'école - et le but recherché par la scolarisation en français - faire de cette langue une langue véritablement seconde, c'est-à-dire apte à remplir d'autres fonctions.

2. Même en restant au niveau des seuls besoins langagiers, il n'est pas sûr que les priorités du français de scolarisation correspondent bien aux besoins des apprenants. Les résultats de 
l'enquête de la CONFEMEN ${ }^{2}$ sur les besoins langagiers et les centres d'intérêt en français et en langues nationales des élèves du primaire en Afrique francophone sont sur ce point très intéressants : ils montrent la contradiction existant entre ce français de scolarisation et les besoins langagiers des élèves. P. Prévost (1992 : 83-93) présente rapidement ces résultats en les reliant explicitement à la problématique de l'enseignement du FLS. D'une part, il ressort que, pour les enseignants, le français est avant tout la langue de l'école, ce qui semble conforter l'hypothèse de G. Vigner et de J.-P. Cuq ; P. Prévost en conclut donc logiquement qu'il faut développer les interactions dans la classe, au sein de l'univers scolaire. En revanche, s'agissant des besoins langagiers, deux dominantes se dégagent pour les actes de langage, "dire» et "demander». Mais des actes de langage tels qu' "argumenter, comparer, décrire, définir, supposer", essentiels pour la communication scolaire, constitutifs au premier chef d'un éventuel français de scolarisation ne figurent pas en bonne place parmi les besoins langagiers essentiels. On voit donc que, même de ce seul point de vue des besoins langagiers, il n'est pas sûr que le français de scolarisation constitue une réponse appropriée aux attentes des apprenants.

3. Définissant le FLS de façon minimale par la fonction de scolarisation, G. Vigner écrivait également qu'il pouvait «être présent dans l'environnement économique et social des élèves ». Ce trait important, définitoire même, nous semble trop souvent oublié au vu d'autres affirmations qui nient pratiquement tout rôle social au français (cf supra). Pourtant, le français existe souvent en dehors de l'école dans les pays africains et il joue un rôle dans la communication sociale, à l'écrit principalement, rôle qu'il ne faudrait pas trop sousestimer au risque de restreindre encore plus l'usage de cette langue. Il importe de tenir largement compte de ce rôle social dans l'enseignement, ce que les approches communicatives sont à même de faire si on ne les réduit pas à une simple pédagogie de l'oral.

4. Ensuite, "comprendre le sens des enseignements qui sont donnés en français ", pour reprendre les termes de J.-P. Cuq, suppose que l'enfant soit à même de décoder, pour en rester pour l'instant à ce niveau d'exigence, des messages référant à d'autres réalités que les seules réalités scolaires : histoire, géographie, biologie, hygiène, etc. On voit alors mal à quoi peut se ramener cette phase de priorité donnée aux compétences de décodage. Comment les faire acquérir en centrant le discours didactique sur les seules situations d'enseignement? Comment les assurer sans passer par la réutilisation, l'encodage, qui seul peut garantir un investissement effectif des apprenants? La phase de priorité au décodage risque fort, une fois de tels principes mis en pratique, de se réduire à la première semaine de classe au cours de laquelle le maître a à cœur d'apprendre à ses élèves à formuler les actes de langage essentiels à la vie de la classe. Du reste, J.-P. Cuq est bien conscient de cette nécessité d'élargissement langagier puisqu'il propose de «multiplier les situations de communication ». Mais n'est-ce pas là reconnaître implicitement la valeur des méthodes s'appuyant sur les usages sociaux de la langue?

5. Enfin, toutes ces considérations ne tiennent pas assez compte d'une dimension pourtant constitutive de la didactique du FLS: l'âge des apprenants. Or s'il y a véritablement spécificité du FLS par rapport au FLE en matière de didactique, c'est vraiment dans la précocité de cet enseignement: des enfants de cinq, six, sept ans sont confrontés à une langue qu'ils ne connaissent pas et qu'ils doivent comprendre pour pouvoir suivre normalement les cours de mathématiques, histoire, géographie, etc. Il arrive même que l'apprentissage de la lecture doive se faire dans cette langue étrangère : on comprend alors toute la spécificité du concept de FLS et tout l'intérêt d'y consacrer une réflexion particulière. En effet, passé les premières années, la spécificité de l'apprentissage de la langue seconde s'estompe. Peu de choses distinguent alors son étude de celle de la langue maternelle. Quelle est donc la meilleure façon d'enseigner à un tout jeune apprenant les rudiments de la langue? En effet, à l'âge où l'on entre à l'école primaire, sans aucune 
connaissance en français, il ne s'agit pas encore de familiariser les enfants avec les «fonctionnements langagiers spécifiques » au français de scolarisation mais simplement (?) de les faire entrer dans la langue.

Or, nous allons le voir, modèles didactiques ou exemples proposés par les tenants d'un français dit de scolarisation concernent plutôt des publics sensiblement plus âgés.

G. Vigner reconnait certes l'importance de cette précocité, souvent caractéristique des enseignements de FLS (1992: 45). Mais son article est si important, il embrasse des problématiques si vastes (allant des apprentissages précoces au lycée, de l'écolier africain au lycéen madrilène), qu'il ne s'attarde pas à ce niveau de l'apprentissage qui est à nos yeux le plus caractéristique de la majorité des situations didactiques en FLS, particulièrement en Afrique. Traitant par exemple de la lecture, il choisit comme niveau de référence l'articulation primaire/secondaire (1992: 49) et tire quelques principes didactiques intéressants (1992: 51). Mais l'originalité de la problématique de la lecture en FLS est à chercher en amont, dans les premiers moments de la relation didactique, quand l'apprentissage de la lecture doit se faire au plus vite dans une langue encore étrangère.

J.P. Cuq de son côté (1991 : 146) rapporte, après Anh Tran Ngoc, une expérience visant à apporter un remède à l'échec scolaire en Nouvelle-Calédonie. Il est symptomatique que les niveaux concernés par cette réflexion soient ceux des classes du cycle d'observation des collèges (classes de sixième et cinquième). Quand est abordé le problème des débuts de la scolarisation en langue seconde, comme c'est le cas plus loin avec l'exemple de l'enseignement préscolaire dans l'île de Saint-Martin, les propositions rapportées, pour intéressantes qu'elles soient (Martinez, 1987), sont plutôt d'ordre psycholinguistique que réellement méthodologique; elles visent essentiellement à ménager un espace de transition entre espace maternel et scolaire en redonnant leur place aux langues maternelles pendant les « premières acquisitions, la période d'instrumentalisation ». Mais J.-P. Cuq arrête là la citation et rien n'est dit de la manière dont doivent être faits les premiers pas en français. Suit le compte-rendu des expériences d'immersion au Canada, et notamment d'immersion précoce : si les mérites en sont reconnus (prise en compte de la dimension de langue scolaire de la langue seconde, 154 ; «seule tentative didactique véritablement novatrice", 161), les limites sont toujours celles des méthodes communicatives («les méthodes immersives, qui correspondent grosso modo à l' "époque communicative ", semblent donc présenter les mêmes avantages mais aussi les mêmes limites »).

On trouvera toutefois dans l'ouvrage de J.-P. Cuq mention des manuels de français et de lecture en usage en Afrique dans les premières années de l'apprentissage mais sans que ne soit produite une analyse du point de vue de la méthodologie de l'enseignement du français langue seconde (168-188).

Or, le choix de ces exemples de situations didactiques n'est pas à notre avis sans répercussions sur la théorisation que proposent G. Vigner et J.-P. Cuq. Ne posant que rarement la question des tout premiers pas, des premières années d'apprentissage, ces auteurs sont naturellement conduits à mésestimer l'importance des méthodes communicatives, dont le rôle est surtout de permettre à l'apprenant d'« entrer dans la langue ", et à mettre l'accent sur la nécessité de maîtriser les opérations langagières les plus récurrentes dans la communication scolaire.

Ainsi, tout en reconnaissant l'importance de la dimension cognitive du FLS, de sa valeur de langue d'information également, nous pensons que l'on ne peut tout à fait se passer 
des approches communicatives pour enseigner les rudiments de la langue française à de très jeunes enfants. Il faut donc trouver à la didactique du FLS une voie originale alliant le communicatif et la dimension instrumentale. C'est à poser quelques jalons sur cette voie que nous voudrions nous employer maintenant.

\section{4. Éléments pour une didactique du FLS}

Il est, selon nous, primordial de permettre aux jeunes apprenants d'entrer dans la langue seconde en s'investissant dès les premiers instants de la classe, afin de ne pas retomber dans le travers des méthodes d'inspiration audio-orale ou audiovisuelle qui amenaient souvent à produire des énoncés de type constatif à propos d'autres personnes et retardaient trop le moment où l'apprenant pouvait enfin parler de lui et communiquer. En d'autres termes, trop d'énoncés en il ou elle et pas assez en je et tu. De ce point de vue, la volonté de donner la priorité aux compétences de décodage, outre qu'elle nous semble illusoire, ne nous semble pas une option à retenir.

Pour doter les apprenants, dès les premiers moments de la classe, des outils linguistiques leur permettant cet investissement, on peut, conformément aux recommandations des partisans d'un français de scolarisation, trouver de précieux appuis dans les situations de la communication scolaire. La présentation du maître aux élèves, le premier appel présentation minimale des élèves au maitre - sont autant de situations productrices de discours en je et en tu qu'il importe d'exploiter. De manière générale, chaque fois que la vie de la classe le permet, il est bon de s'appuyer sur les situations réelles de communication pour faire progresser la connaissance puis la maîtrise des outils linguistiques et, avec elle, de la compétence de communication. L'ordre, l'interdiction, l'interrogation, la définition, sont des situations langagières très fréquentes qu'il faut bien sûr maîtriser d'abord en classe avant de pouvoir les transférer dans la vie sociale.

Mais il ne faut pas pour autant se cantonner à ces situations scolaires de communication pour assurer les débuts de l'apprentissage. D'une part, cela contribuerait à couper encore plus l'école de la société ; d'autre part, une telle démarche n'aurait que peu de chances de rencontrer l'intérêt des enfants, qui aiment à retrouver en classe des situations de la vie de tous les jours; enfin ce serait méconnaître les besoins en imaginaire de petits enfants qui n'aiment rien tant que jouer, inventer, faire comme si. S'agissant de très jeunes apprenants, un des atouts majeurs des méthodes communicatives est sans doute la place accordée au jeu de rôle dans l'apprentissage de la langue. La préparation du jeu de rôle est l'occasion d'une exploration linguistique en même temps que d'une prise de conscience des contraintes ethnosocioculturelles qui pèsent sur la communication; sa réalisation permet à l'apprenant de mettre tout cela en pratique. Le jeu de rôle permet surtout au jeune enfant de jouer véritablement; il le place dans un monde où il doit faire comme si, ce qu'il est très largement habitué à faire au cours de ses propres jeux. Dès lors, dans un univers ludique, peu importe que le français soit pratiqué dans des situations dans lesquelles les langues maternelles ou un autre véhiculaire auraient normalement cours : du moment que tout est fictif, que l'enfant est déjà dans la peau d'un autre personnage, pourquoi vouloir à tout prix coller à la réalité sociolinguistique des échanges langagiers? Cela choque plus les observateurs adultes que les enfants, habitués à se mouvoir dans des univers de fiction constitués selon des règles différentes. En fait, le jeu de rôle, au départ conçu pour des méthodes destinées à des publics adultes, s'insère tout naturellement dans l'apprentissage des plus jeunes. 
32 Au niveau individuel, la communication ainsi simulée peut permettre à l'enfant d'apprivoiser peu à peu, depuis l'école, les rites de communication de sa société et de le faire sans risque dans un univers imaginaire. Au niveau social, ce type d'exercice peut contribuer à rapprocher un peu plus l'école de la société, ou tout au moins à ne pas trop marquer la coupure qui existe trop souvent.

Pour donner un exemple de l'utilité des approches communicatives dans ces premiers instants de l'apprentissage, nous choisirons le domaine de la lecture. Les apprenants scolarisés en français sont le plus souvent des enfants issus de sociétés ou de milieux sociaux dans lesquels ils ne sont qu'exceptionnellement confrontés à l'écrit. Avant de commencer l'apprentissage de la lecture, quelle que soit la méthodologie choisie, il importe de faire comprendre à l'enfant la fonction, l'utilité, de la chose écrite. Il faut créer le besoin de lire en montrant l'utilité de la lecture dans la société et pas seulement dans l'univers scolaire. Pour ce faire, il faut commencer par introduire dans l'univers de la classe des écrits sociaux qui sont, dans beaucoup de pays où le français est langue seconde, en français. Ces textes seront replacés dans une situation de lecture, sinon vraiment authentique du moins simulée, au moyen d'un jeu de rôle permettant d'en faire saisir la fonction, de montrer aux élèves à quoi sert l'écrit et donc pourquoi il faut apprendre à lire. Communications orale et écrite sont donc-menées de front. On commencera par des textes très courts - un mot suffit pourvu qu'il soit réellement porteur d'un message - dont le sens pourra être déduit facilement de la situation de communication, et qui pourront être reconnus de manière globale. Ainsi, dans les premiers mois de l'apprentissage, les apprenants se constitueront un stock de mots suffisant pour passer ensuite à une deuxième phase, plus classique, consacrée à l'analyse et à la combinatoire des unités ainsi dégagées - groupes de lettres puis lettres ${ }^{3}$. L'apprentissage de la lecture, fondamental pour l'enseignement d'une langue de scolarisation, gagne donc à être mené en tenant compte des principes et des méthodes communicatifs, qui permettent de faire prendre conscience de l'utilité de l'écrit avant d'en commencer l'étude. Ce n'est que dans un troisième temps qu'il faut, conformément à ce que proposent G. Vigner et J.-P. Cuq, insister sur la dimension d'information de la langue française, moyen d'accession à la connaissance dans toutes les matières scolaires. En lecture, suivant en cela les travaux récents sur la typologie textuelle, on pourra faire porter l'accent sur le décodage des unités qui assurent la transmission d'une information; ainsi pourra-t-on privilégier la lecture de textes de type explicatif ou informatif, très fréquents dans le discours scolaire et vecteurs du savoir dans toutes les disciplines.

On le voit, la tâche est complexe; la démarche retenue doit allier les dimensions communicative (pour les débuts de l'apprentissage, pour faire entrer de plain pied l'enfant dans la langue) et de scolarisation, d'information (pour la suite du parcours scolaire).

\section{FLS et public non-francophone en France}

Toute cette réflexion sur le FLS, encore très récente aujourd'hui, prend naissance dans des terrains sociolinguistiques où le français, langue de l'école, entre en contact avec des langues maternelles dont le statut, à divers degrés minoré, les empêche d'accéder à cet univers moderne de la transmission des savoirs. Les pays d'Afrique, anciennes colonies françaises, sont des aires de recherche importantes dans lesquelles les problèmes de 
didactique du français se doublent bien souvent de questions de pédagogie liées à l'importance des effectifs, à la pauvreté des moyens disponibles, etc.

Pour autant, ces travaux ne sont pas sans devoir intéresser directement l'enseignant de français en France, à qui il arrive d'enseigner à de très jeunes migrants pour lesquels le français n'est pas langue maternelle. Il est vrai, comme l'avance à juste titre J.-P. Cuq (1991: 140), que ce public ne saurait être tout à fait assimilé à un public de FLS de type africain, en raison du fait que l'issue visée de l'apprentissage est une assimilation et un monolinguisme pour les premiers, un bilinguisme pour les seconds. Il n'en demeure pas moins que, si l'on considère la scolarisation des jeunes migrants en début de primaire, ceux-ci rencontrent exactement les mêmes problèmes d'apprentissage: ils doivent apprendre à lire dans une langue encore étrangère, recevoir en français le savoir dispensé dans toutes les disciplines, apprendre à maitriser au plus vite les situations de communication scolaires et extrascolaires.

Les directions de travail que nous avons pu avancer dans la dernière partie de notre réflexion en matière de didactique restent donc valables pour l'essentiel. Non seulement les méthodes d'inspiration communicative peuvent donc aussi jouer un rôle positif, mais les arguments avancés contre leur emploi en situation africaine n'ont plus aucune raison d'être: alors que l'on était en droit de redouter une contradiction avec les rituels de communication africains, n'accordant qu'une faible importance à la libre expression de l'enfant, la place toujours croissante faite à l'enfant dans la société française légitime ces méthodes " actives »; alors qu'il n'y avait pas nécessité d'apprendre à communiquer en français quand ce rôle était très bien rempli par les langues maternelles, l'intégration à la société d'accueil commande d'apprendre au plus vite à se servir du français dans tous les échanges sociaux, dès la sortie du milieu familial. Le recours à ces méthodes s'en trouve donc comme légitimé par surcroît ...

\section{Conclusion}

L'acquisition des matériaux linguistiques, chez de très jeunes enfants, gagne à l'utilisation de méthodes communicatives. Même si les jeux de rôle en français ne correspondent pas tous à une réalité des échanges, ils ont le mérite de rejoindre les centres d'intérêt des enfants et d'être des situations-problèmes permettant d'explorer des situations de communication et d'acquérir les structures langagières et le vocabulaire nécessaires pour parler de la classe mais aussi d'eux-mêmes et de leur environnement. Les premiers instants de la classe de langue pourront s'appuyer sur la communication scolaire, le monde de la classe : les rudiments du français seront acquis dans ce cadre authentique de communication; puis, on élargira l'univers référentiel de l'apprentissage en pratiquant une communication simulée à l'aide des jeux de rôles. Enfin, l'apprentissage du français gagnera à privilégier l'aspect informatif de la langue seconde, à explorer les formulations, les structures, récurrentes dans une langue qui sert avant tout - mais pas seulement ... - à transmettre des connaissances, à véhiculer le savoir scolaire.

Fonder la didactique du FLS sur la dimension de scolarisation est donc une idée intéressante, mais elle n'est rentable que pour les toutes premières semaines de l'apprentissage (le temps d'acquérir les rudiments de la communication nécessaires à la vie de la classe) ou pour des niveaux déjà avancés (cycle des approfondissements, au minimum, pour exercer des capacités de lecture et d'écriture nécessaires à la réussite 
scolaire). Mais pour l'essentiel du cycle des apprentissages, les méthodes communicatives restent les plus proches des centres d'intérêt des enfants, les plus aptes à étendre leurs compétences linguistiques et celles qui contribuent le mieux à relier l'univers scolaire au reste de la société.

\section{BIBLIOGRAPHIE}

BARBE G., « Français langue seconde : attention travaux », Diagonales. n 14, 1988.

BESSE H., NGALASSO M. M., VIGNER G., (coord.), « Français langue seconde », Études de Linguistique Appliquée, $\mathrm{n}^{\circ}$ 88, 1992.

CORNETTE Y., DAOUD Z. H., MAURER B., ROBERT J.-P., Le cabri et le chacal. Classe d'initiation. EDICEF, Paris, 1993.

CORNETTE Y., DAOUD Z. H., MAURER B., ROBERT J.-P., Les belles histoires de Saïd et Saïda. Cours préparatoire. EDICEF, Paris. À paraître, 1994.

CUQ J.-P., Le français langue seconde. Origines d'une notion et implications didactiques. Hachette, Paris, 1991.

CUQ J.-P., « Français langue seconde. Un point sur la question » in BESSE H., NGALASSO M. M., VIGNER G., (coord.), Français langue seconde, Études de Linguistique Appliquée, nº 88, 1992. Pages $5-26$.

CALISSON R., COSTE D., Dictionnaire de didactique des langues. Hachette, Paris, 1976.

NGALASSO M. M., « Le concept de français langue seconde », in BESSE H., NGALASSO M. M., VIGNER G., (coord.). Français langue seconde. Études de Linguistique Appliquée, nº 88, 1992. Pages 27-38.

ROULET E., « Description du français contemporain et didactique » (1970-1985), dans Études de Linguistique appliquée, $\mathrm{n}^{\circ}$ 63, 1986.

ROULET E., « L'apport des sciences du langage à la diversification des méthodes d'enseignement des langues secondes en fonction des caractéristiques des publics visés ", in Études de Linguistique appliquée, $\mathrm{n}^{\circ} 21,1975$.

ROULET E., Langues maternelles et langues secondes, vers une pédagogie intégrée, Paris, Hatier-CREDIF, 1980.

VIGNER G., « Français langue seconde : une discipline spécifique », dans Diagonales, n 4, 1987. Pages 42-45.

VIGNER G., « Le français langue de scolarisation » in BESSE H., NGALASSO M. M., VIGNER G., (coord.). Français langue seconde, Études de Linguistique Appliquée, nº 88, 1992. Pages 39-53. 


\section{NOTES}

1. On trouve aussi l'expression de langue d'information (Vigner, 1992: 43): c'est par cette scolarisation que s'organisent les connaissances, se mettent en forme les données de l'expérience. Par là, la langue seconde devient langue de développement, la fonction d'information «permettant à l'enfant de construire progressivement des représentations d'objets » (Vigner, $1987: 45)$.

2. Conférence des Ministres de l'Éducation Nationale des pays ayant en commun l'usage du français.

3. Cette approche est celle qui a été retenue pour la création d'une nouvelle méthode d'enseignement du français en République de Djibouti. Cornette (Y.), Daoud (Z. H.). Maurer (B). Robert (J.-P.). Le cabri et le chacal. Classe d'initiation. EDICEF, Paris, 1993 et Les belles histoires de Saïd et Saïda. Cours préparatoire. EDICEF, Paris. 1994.

\section{RÉSUMÉS}

Prenant en compte les propositions récentes de plusieurs chercheurs en matière de didactique du français langue seconde, l'auteur s'efforce de penser la spécificité didactique de cette situation linguistique et examine de manière critique les propositions faites pour promouvoir une didactique du FLS en termes de français de scolarisation. Il essaie d'en faire ressortir les avantages, notamment d'ordre cognitif. Mais la prise en compte du terrain d'Afrique francophone l'amène à mesurer les limites d'une telle approche, pas assez adaptée à la scolarisation de très jeunes enfants dans une langue qui leur est étrangère. Il cherche donc des pistes visant à concilier approches communicatives et français de scolarisation. À la fin de l'article, quelques réflexions situent ce débat, né dans un champ didactique situé hors de France, dans la perspective de l'enseignement du français pour enfants non francophones en France.

Taking into account the recent propositions of several researchers on the discipline of the didactics of french as a second language, the author endeavors to think through what is didactically specific to this linguistic situation and in a critical fashion examines the proposal made towards promoting a didactic of french as a second language defined in terms of classroom french. He attempts to point out the advantages of such a method, particularly from a cognitive stand-point. But, the taking into account of the situation in the field in french speaking regions of Africa brings him to take the measure of the limits of such an approach, insufficiently adapted to the schooling of very young children in a language which is foreign to them. He therefore researches lines of thought aiming at harmonising communicational approaches and classroom french. At the end of the article, as this didactic study originates in an area located outside of France, a few reflections put this discussion into its context, i.e., within the perspective of the teaching of french to non french speaking children in France. 
INDEX

Mots-clés : Afrique francophone, didactique du FLE, didactique du FLS, français de scolarisation, public migrant

Keywords : classroom french, didactic of french as a Foreign Language (FFL), Didactic of French as a second language (FSL), french speaking Africa, Migrant population

\section{AUTEUR}

\section{BRUNO MAURER}

Maître de conférences en Sciences du langage, directeur d'études, IUFM de Montpellier 